Research Article

Open Access

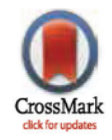

${ }^{1}$ Chemistry Department, College of Science, Sudan University of Science and Technology, Sudan.

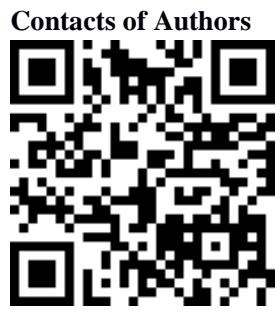

* To whom correspondence should be addressed: Mohammed Sulieman Ali Eltoum

Citation: Eltoum M S A, Elfaki A A M (2020). Extraction, characterization, and usage of Turmeric Curcumin for color coating of metronidazole tablets. Highlights in BioScience Volume 3. Article ID 20206. dio:10.36462/ H.BioSci.20206

Received: May 20, 2020

Accepted: June 27, 2020

Published: June 30, 2020

Copyright: () 2020 Eltoum and Elfaki .This is an open access article distributed under the terms of the Creative Commons Attribution License, which permits unrestricted use, distribution, and reproduction in any medium, provided the original author and source are credited.

Data Availability Statement: All relevant data are within the paper and supplementary materials

Funding: The authors have no support or funding to report.

Competing interests: The authors declare that they have no competing interests.

\section{Extraction, Characterization, and Usage of Turmeric Curcumin for Color Coating of Metronidazole Tablets}

\author{
Mohammed Sulieman Ali Eltoum*1 ${ }^{*}$, ALMustafa Ahmed Mohamed Elfaki ${ }^{1}$
}

\section{Abstract}

Curcumin is a chemical compound produced by Curcuma longa plants that is widely used as a coloring agent and a dietary supplement and has some therapeutic activity. Our aim is to evaluate the use of curcumin as color coating material for metronidazole tablets. Curcumin was extracted at higher yields from three different samples of turmeric plants. The extract obtained was characterized by Infrared Red Spectroscopy (IR), Thin Layer Chromatography (TLC) and ash content and melting point (MP). The curcumin produced has an MP content ranging from $182^{\circ} \mathrm{C}$ to $184^{\circ} \mathrm{C}$ and an ash content ranging from 1.5 to $3.17 \%$. Curcumin material was used as a colorful agent for the coating of metronidazole tablet pills using spray coating technology. Experimental results have shown that curcumin-coated metronidazole tablets exhibit strong color stability even at higher temperatures, and that the taste of bitterness in metronidazole pills has been reduced or has disappeared entirely.

Keywords: Curcumin, Turmeric, Color-coating, Metronidazole tablets, Pharmaceutical.

\section{Introduction}

Scientific research on color chemistry is needed to improve and maintain color stability. Natural coloring additives are generally considered to be coloring additives derived from plant or animal sources by extraction or other physical processing[1,2]. Examples of natural colorants include carmine, annatto extract, grape skin extract, turmeric, saffron and beta-carotene, which are the major natural color additives used in food products. Synthetic coloring additives include chemically synthesized substances such as tartrazine, erythrosine and indigo carmine. Natural or vegetable colors are less in use because they are expensive or difficult to extract $[1,2]$. Curcumin (synonyms: turmeric yellow, kurkum, INS No. 100(i)) is an orange-yellow crystalline powder.

The standards of the Joint Expert Committee on Food Additives (JECFA) define only curcumin extracted from natural source materials. It may also be produced by chemical synthesis, which is not used as a food additive [3]. The chemical structure of curcumin is shown in Figure 1. The yellow coloring components (curcuminoids) and the primary coloring pigment are curcumin (CurI), along with two additional minor curcuminoids, demethoxycurcumin (CurII) and bisdemethoxycurcumin (CurIII), which are the key active constituents of the turmeric (Figure 2). Curcumin is a major secondary metabolite of the perennial Asian plant turmeric (Curcuma longa L). Curcumin was identified as the active principle of turmeric in 1815 and its structure was determined after crystallization in 1870 [4]. Turmeric is only one representative of more than 80 species of turmeric in the ginger family, Zingiberaceae [5]. 


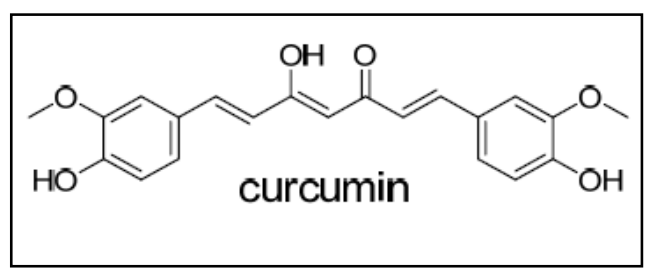

Figure 1: The chemical structure of curcumin.

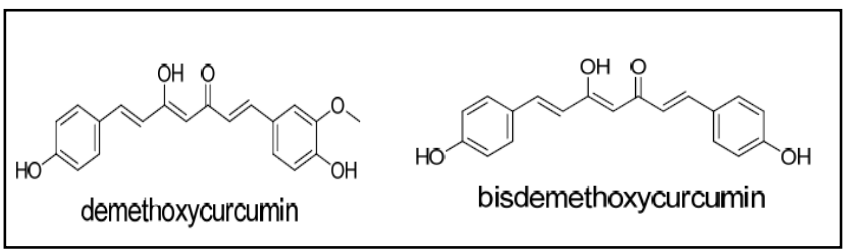

Figure 2: Structure of the minor curcuminoids.

Turmeric is widely cultivated in many Asian countries, particularly India, where it is mainly grown for dietary use and is a major component of the spice curry. In addition, turmeric is recognized for its medicinal properties and has been used for centuries in the treatment of a variety of diseases including eczema, arthritis, ulcers, asthma, anemia and many others [6]. As a result of extensive studies over the last few decades, curcumin has emerged as a promising anti-cancer agent and has been shown to target multiple and diverse pathways of disease causation and progression [4]. The attractiveness of curcumin as a therapeutic agent is enhanced by its safety, affordability, and history of longterm use [7]. Molecular formula of C21H20O6, corresponding to a molecular weight of 368.37 .

Curcumin is a yellow-orange crystalline powder with maximum absorbance at430 nm and melting point of $183{ }^{\circ} \mathrm{C}$ [6]. Curcumin exhibits hydrophobic and (slight) hydrophilic properties owing to its aliphatic heptadienone linker and polar $\beta$-dicarbonyl and phenolic groups, respectively [8]. Curcumin is sparingly soluble in water, but shows greater solubility in some organic solvents such as acetone, ethyl acetate, acetonitrile and ethanol. Its reported partition coefficient $(\log \mathrm{P})$ ranges from 2.5 to 3.3 [9]. Curcumin is a bis- $\alpha, \beta$-unsaturated $\beta$ - diketone and exists in equilibrium with its enol tautomer [10]. Studies involving 1H, C NMR, and infrared spectroscopy have shown that the enolate form predominates in alkaline solution [11].

No previous literature has introduced the use of curcumin as a color coating for metronidazole tablets. The aim of this study is to extract and characterize curcumin obtained from the turmeric plant using different analytical techniques and to evaluate the use of turmeric powder as a coating material for metronidazole tablets. In addition, we studied the effect of this coat material on some of the properties of the metronidazole tables.

\section{Materials and Methods}

Turmeric samples were collated from Sudanese local market.

Curcumin extraction: Twenty grams of ground turmeric in $50 \mathrm{~mL}$ of dichloromethane was magnetically stirred and heated at the reflux condenser for $1 \mathrm{~h}$. The mixture was then suction-filtered and the filtrate was concentrated in a hot water bath maintained at $50^{\circ} \mathrm{C}$. The reddish yellow oily residue was treated with $20 \mathrm{ml}$ hexane and the resulting solid was collected by suction filtration [3]. The obtained yield was calculated as follow: Yield $=\mathrm{Wpr} / \mathrm{W}$ theoX100 Where: $\mathrm{Wpr}=$ practical weight, $\mathrm{W}$ theo $=$ theoretical weight

Fourier Transform Infrared Spectroscopy (FT -I R): Two milligrams of curcumin were mixed with $300 \mathrm{mg}$ of dried potassium bromide. Carefully, the mixture was grinded, spread evenly in an appropriate die and subjected to a pressure of approximately $800 \mathrm{MPa}\left(8 \mathrm{t} \cdot \mathrm{cm}^{-2}\right)$. In the case of substances that were unstable under normal atmospheric conditions, the disc was pressed into a vacuum. A disc was rejected if visual examination had been show lack of uniform transparency or when transmittance at 2000 $\mathrm{cm}^{-1}(5 \mu \mathrm{m})$ in the absence of a specific absorption band was less than 60 per cent without compensation, unless otherwise prescribed. Samples were prepared by the same procedure and the spectrum was recorded between $4000-400 \mathrm{~cm}^{-1}$ (2.5-15.4 $\mu \mathrm{m})$ under the same operational conditions. The transmission minima (absorption maxima) in the spectrum was obtained with the substance to be examined correspond in position and relative size to those in the spectrum obtained with the reference substance [12].

Thin Layer Chromatography (TLC): The solvent system which was used to characterize of curcumin powder was prepared by mixed of $(3 \%$ methanol and $97 \%$ dichloromethane). The diameter of TLC plate had 10X20 $\mathrm{cm}^{2}[3]$.

Melting point (MP): A sufficient quantity of curcumin was introduced into the capillary tube to give a compact column of $4 \mathrm{~mm}$ to $6 \mathrm{~mm}$ in height. The temperature of the bath was increased to about $10^{\circ} \mathrm{C}$ below the assumed melting point and the heating rate was adjusted to about $1^{\circ} \mathrm{C} / \mathrm{min}$. When the temperature was $5^{\circ} \mathrm{C}$ below the assumed melting point, the capillary tube was introduced into the instrument. In the case of the apparatus described above, the capillary tube was immersed in such a way that the closed end is near the center of the thermometer bulb, the immersion mark of which is at the surface level of the liquid. The temperature at which the last particle was transferred to the liquid phase was recorded [12]. 
Ash content: The porcelain crucible was ignited at $600 \pm 50$ ${ }^{\circ} \mathrm{C}$ for $30 \mathrm{~min}$ and then allowed to cool over the silica gel in the desiccators. The prescribed amount of curcumin was placed in the crucible and weighed, after it had been gently heated to low temperatures as practicable, until the sample had been thoroughly charred, until white fumes had ceased to develop and ignited at $600 \pm 50{ }^{\circ} \mathrm{C}$ until the residue had been completely incinerated. The crucible was allowed to cool over the silica gel in the desiccator, was weighed again and the percentage yield was calculated [12].

Percentage of whiteness: The sample powder was placed in dishes, regularly highlighted and then the color was measured against the white color standard and the percentage was finally recorded.

Coating of metronidazole tablets with curcumin: A total of $25 \mathrm{~g}$ of Opadry white was dissolved in $500 \mathrm{ml}$ of ethanol with stirring during $30 \mathrm{~min}$. and $1 \mathrm{~g}$ of curcumin powder was added during $10 \mathrm{~min}$. The spray coating process for metronidazole tablets began by placing the tablets in the coating machine chamber, and then the main unit button was pressed.The exhaust was operated until the powder was removed from the tablets and the hot air was operated until the temperature of the tablet reached $50^{\circ} \mathrm{C}$. The temperature control key was operated with hot air to control the temperature of the coating. The spray stage was then operated, and the turn sprayers were opened until the tablets were completely coated. And after coating, the other keys were turned off until the tablets had dried. The coating machine was stopped; the tablets were removed [12].

\section{Results and Discussion}

The turmeric plant can be identified both by its characteristic tuberous root and by the leaves which extend upward from the erect, thick stems of the root. Turmeric root has a fragrant aroma and a slightly bitter, peppery, biting taste reminiscent of ginger. When eaten, it colors the saliva yellow and leaves a warm feeling in the mouth. The curcumin yields obtained for the different samples are shown in Table 1 and the resulting compounds were finely soft, clear yellow powder. The yield of the samples was consistent with the method described by Andrew et al, [3], and the appearance of the powder was as clear as the standard colour.

Table 1: The Curcumin yield (CY), Melting point (MP), Ash Content (AC) and Percentage of Whiteness (PW) of the studied tumeric samples.

\begin{tabular}{ccccc}
\hline $\begin{array}{c}\text { Samples } \\
\text { Number }\end{array}$ & CY & MP & AC & PW \\
\hline $\mathbf{1}$ & $86.36 \%$ & $183^{\circ} \mathrm{C}$ & $1.50 \%$ & $52.04 \%$ \\
$\mathbf{2}$ & $81.81 \%$ & $184^{\circ} \mathrm{C}$ & $3.09 \%$ & $53.03 \%$ \\
$\mathbf{3}$ & $82.72 \%$ & $182^{\circ} \mathrm{C}$ & $3.17 \%$ & $51.08 \%$ \\
\hline \hline
\end{tabular}

Figures 3 show the absorption of samples. These Figures contain the following characteristic peaks: the broad band of the three $\mathrm{OH}$ groups at $3250-3500 \mathrm{~cm}^{-1}$, sharp peak Stretch of $\mathrm{C}$ - $\mathrm{H}$ aliphatic system less than $3000 \mathrm{~cm}^{-1}$, weak peak of $\mathrm{C}-\mathrm{H}$ aromatic system around $3050 \mathrm{~cm}$, sharp peak of $\mathrm{C}=\mathrm{O}$ at $1510 \mathrm{~cm}^{-1}$, medium peak of $\mathrm{C}-\mathrm{O}$ at $1300 \mathrm{~cm}^{-1}$, and sharp peak of $\mathrm{C}=\mathrm{C}$ of aromatic system at about $1510 \mathrm{~cm}^{-1}$. Figure 4 shows the TLC plate of the compound. The chromatogram obtained showed that curcumin was divided into three distinct compounds. Clearly, this result is in agreement with Andrew et al., [3].

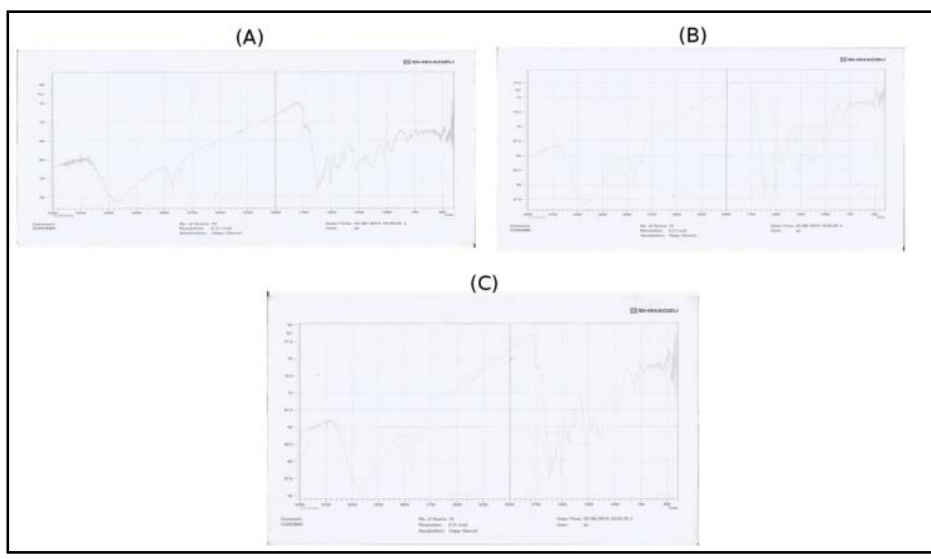

Figure 3: IR spectrum analysis results of curcumin for samples 1 (A), 2 (B) and 3 (C).

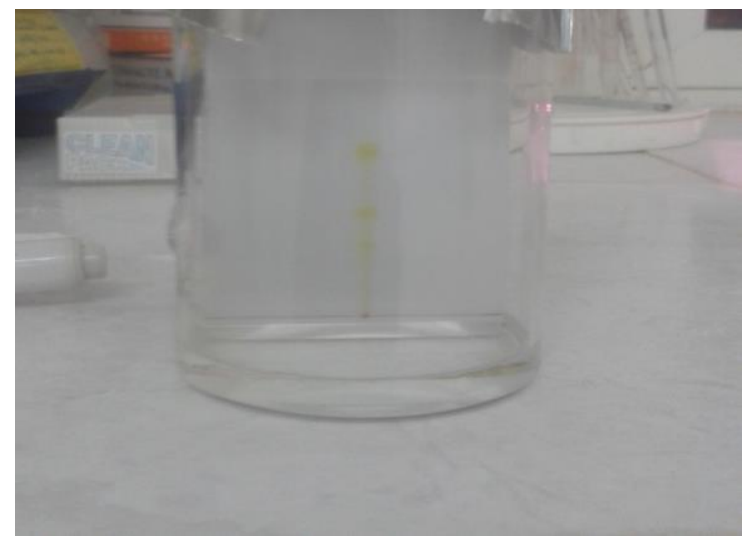

Figure 4: TLC of curcumin sample.

The melting point determined by the capillary method and it is the temperature at which the last solid particle of a compact column of a substance in a tube passes into the liquid phase. The results of the three samples are shown in Table 1. The melting point of curcumin is $183^{\circ} \mathrm{C}$ (BP2012)[12]. The ash content obtained of the samples ranges from 1.5 to $3.17 \%$ (Table 1). In summary, from the three samples above, the percentage of Ash in curcumin was good. The percentage of whiteness of the three samples obtained ranges from $51.08 \%$ to $53.03 \%$ (Table 1).

Coated tablets are tablets containing one or more layers of mixtures of different substances, such as natural or synthetic resins, gums, gelatin, inactive and insoluble fillers, sugars, plasticizers, polyols and waxes. Coloring matter authorized by the competent authority and sometimes flavorings and 
active substances. The mean weight and disintegration time of metronidazole tablets prior to coating with curcumin was $323.0 \mathrm{mg}$ and $23 \mathrm{sec}$ and $22 \mathrm{sec}$ respectively. However, the average weight and disintegration time after coating was $324.74 \mathrm{mg}$ and $120 \mathrm{sec}, 93 \mathrm{sec}$ and $114 \mathrm{sec}$ respectively (Figure 5).

(A)
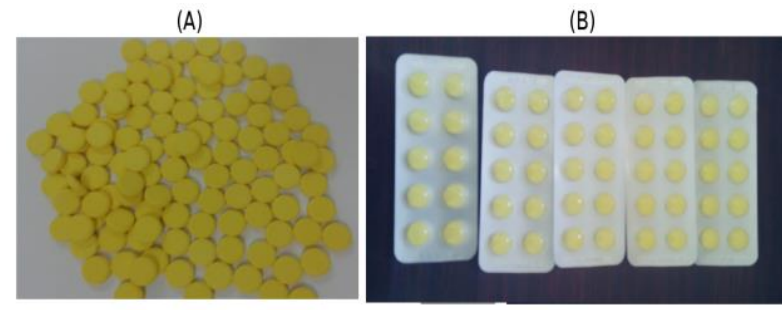

Figure 5: Metronidazole tablets before (A) and after blistering.

\section{Conclusion}

The primary purpose of the study in this study is to study the isolation of curcumin from turmeric and also to characterize curcumin as a color-coating martial for metronidazole tablet in pharmaceuticals. Curcumin was extracted from the turmeric in a simple and easy way using dichloromethane and triturated with hexane, and the extraction yield was finely soft yellow powder. Experimental results have shown that curcumin-coated metronidazole tablets exhibit strong color stability even at higher temperatures, and that the taste of bitterness in metronidazole pills has been reduced or has disappeared entirely.

\section{Acknowledgments}

We would like to thank the Climax Factory for the Drugs and Medicines Industry-Khartoum for giving us the opportunity to carry out the practical work of this research in their different laboratories, and we would also like to thank the Sudan University of Science for accepting this work as part of the complementary research on the requirements of the M.Sc. in Chemistry [13].

\section{References}

1. Allen L, Ansel HC. Ansel's pharmaceutical dosage forms and drug delivery systems. Lippincott Williams \& Wilkins; 2013 Dec 23.

2. Carter SJ. Cooper and Gunn's Dispensing for Pharmaceutical Students (12th Edn) Publisher. 47-50

3. Anderson AM, Mitchell MS, Mohan RS. Isolation of curcumin from turmeric. Journal of chemical education. 2000 Mar;77(3):359

4. Aggarwal BB, Harikumar KB. Potential therapeutic effects of curcumin, the anti-inflammatory agent, against neurodegenerative, cardiovascular, pulmonary, metabolic, autoimmune and neoplastic diseases. The international journal of biochemistry \& cell biology. 2009 Jan 1; 41(1): 40-59.

5. Leong-Škorničková J, Šída O, Jarolímová V, Sabu M, Fér T, Trávníček P, Suda J. Chromosome numbers and genome size variation in Indian species of Curcuma (Zingiberaceae). Annals of Botany. 2007 Sep 1; 100 (3): 505-26.

6. Goel A, Kunnumakkara AB, Aggarwal BB. Curcumin as "Curecumin": from kitchen to clinic. Biochemical pharmacology. 2008 Feb 15; 75 (4):787-809.

7. Chandran B, Goel A. A randomized, pilot study to assess the efficacy and safety of curcumin in patients with active rheumatoid arthritis. Phytotherapy research. 2012 Nov; 26 (11):1719-25.

8. Balasubramanian K. Molecular orbital basis for yellow curry spice curcumin's prevention of Alzheimer's disease. Journal of agricultural and food chemistry. 2006 May 17; 54 (10):3512-20.

9. Grynkiewicz G, Ślifirski P. Curcumin and curcuminoids in quest for medicinal status. Acta Biochimica Polonica. 2012 May 14; 59 (2).

10. Chignell CF, Bilskj P, Reszka KJ, Motten AG, Sik RH, Dahl TA. Spectral and photochemical properties of curcumin. Photochemistry and photobiology. 1994 Mar; 59(3):295-302.

11. Sun YM, Wang RX, Yuan SL, Lin XJ, Liu CB. Theoretical study on the antioxidant activity of curcumin. Chinese Journal of Chemistry. 2004 Aug; 22(8):827-30.

12. BP. The British pharmacopoeia 2012.

13.http://repository.sustech.edu/handle/123456789/13398?sh ow $=$ full 